Iustitia Socialis. Revista Arbitrada de Ciencias Jurídicas.

Año IV. Vol. IV. N 1 . Edición Especial 2019

Hecho el depósito de Ley: FA2016000064 ISSN: 2542-3371

FUNDACIÓN KOINONIA (F.K). Santa Ana de Coro, Venezuela

Tania Muñoa Vidal

http://dx.doi.org/10.35381/racji.v4i1.541

\title{
Una mirada a la culpabilidad de las personas naturales en el Código Orgánico Integral Penal
}

\section{A look at the guilt of natural persons in the Organic Comprehensive Criminal Code}

\author{
Tania Muñoa Vidal \\ tmunoa@sangregorio.edu.ec \\ Universidad San Gregorio de Portoviejo, Manabí \\ Ecuador \\ https://orcid.org/0000-0003-4820-9666
}

Recibido: 30 de octubre de 2019

Aprobado: 03 de diciembre de 2019

\section{RESUMEN}

Se realiza una revisión sobre la culpabilidad, como categoría dogmática que se funde en la Teoría del Delito; y, que se asume por la codificación penal ecuatoriana, sobre los escenarios de la responsabilidad penal, de la imputabilidad y del conocimiento de la antijuridicidad; lo que se contextualiza a partir de las causas de inculpabilidad que se prevén y determinan como el trastorno mental, la edad en personas menores de 18 años de edad, y la responsabilidad en caso de embriaguez o intoxicación.

Descriptores: Culpabilidad; responsabilidad penal; imputabilidad; trastorno mental; edad; embriaguez e intoxicación.

\section{ABSTRACT}

A review of guilt is carried out, as a dogmatic category that is based on the Theory of Crime; and, which is assumed by the Ecuadorian criminal codification, on the scenarios of criminal responsibility, imputability and knowledge of unlawfulness; what is contextualized from the causes of inculpability that are foreseen and determined as the mental disorder, the age in persons under 18 years of age, and the responsibility in case of intoxication or intoxication.

Descriptors: Culpability; criminal liability; imputability; metal disorder; age; drunkenness and intoxication 
Iustitia Socialis. Revista Arbitrada de Ciencias Jurídicas.

Año IV. Vol. IV. N 1 . Edición Especial 2019

Hecho el depósito de Ley: FA2016000064 ISSN: 2542-3371

FUNDACIÓN KOINONIA (F.K). Santa Ana de Coro, Venezuela

Tania Muñoa Vidal

\section{INTRODUCCIÓN}

El Derecho Penal en su Parte General, desarrolla la Teoría del Delito, desde la cual se explica como pieza angular la conducta humana con relevancia jurídica penal, y que se estructura en tres categorías dogmáticas: la tipicidad la antijuridicidad y la culpabilidad, siendo éste último escenario el que se trata en el presente trabajo, en el ámbito de la responsabilidad penal; $y$, desde la aptitud, capacidad y facultades psíquicas del autor, pues como expresa Muñoz (2012) en su obra Derecho Penal Parte General:

Tras un minucioso análisis del Derecho Penal positivo, la Dogmática jurídico-penal ha llegado a la conclusión de que el concepto de delito responde de una doble perspectiva que, por un lado, se representa a) como un juicio de desvalor que recae sobre la conducta; $y$, por otro, b) como un juicio de desvalor que se hace sobre el autor de ese hecho. Al primer juicio de desvalor se le llama ilicitud o antijuricidad. Al segundo culpabilidad o responsabilidad.

Es decir, en la culpabilidad se trata de atribuir el desvalor del hecho típico y antijurídico a su autor. En la República de Ecuador, bajo la promulgación del Código Orgánico Integral Penal, el artículo 34, referente a la culpabilidad, expresa: "Para que una persona sea considerada responsable penalmente deberá ser imputable y actuar con conocimiento de la antijuridicidad de su conducta". De ello se deduce una triada de elementos trascendentales que devienen en la estructura de la culpabilidad:

a) Responsabilidad jurídica;

b) Imputabilidad;

c) Conocimiento de la antijuridicidad.

La responsabilidad jurídica, es el primer elemento que se deduce de lo anterior; y, se traduce en el deber de asumir las consecuencias previamente establecidas de forma legal por la realización de un hecho antijurídico, empero ello no es suficiente al tratarse de responsabilidad jurídica penal, pues en ella habría que añadir que el sujeto que realiza la conducta penalmente relevante, es imputable, es decir, es capaz de soportar las consecuencias jurídicas penales de su acción u omisión, que no sería otra que la pena o sanción. Según el catedrático español Mir Puig (2011), en su obra Derecho Penal Parte General: 
La infracción personal de una norma primaria de determinación permite imputar la antijuridicidad penal a su autor, pero ello no basta para considerar adecuada la imposición al mismo de una pena. Esta no recae directamente sobre el hecho, sino sobre su autor, de modo que para que resulte legítima no basta un hecho penalmente antijurídico y concretamente antinormativo, sino que es preciso que su autor aparezca como un sujeto idóneo para responder penalmente. He aquí el significado de la responsabilidad penal del sujeto como último presupuesto de un hecho punible a su autor.

Un segundo elemento significativo sería la imputabilidad, entendido como la capacidad que exige el Derecho Penal al sujeto trasgresor de la norma prohibitiva, conminada bajo una consecuencia penal, para que el mismo pueda encarar esas consecuencias, es decir, pueda responder en el orden jurídico penal. De lo anterior se infiere que resulta una condición sine qua non la existencia de la imputabilidad para que exista responsabilidad jurídica penal y que esta requiere de dos elementos:

a) Capacidad de comprender lo injusto del hecho

b) Capacidad de dirigir la actuación conforme a dicho entendimiento

Como último elemento de la triada, es necesario además, que el autor actúe con conocimiento de la antijuridicidad de su conducta, particular que aun muy debatido, desde su ubicación sistémica dentro de las categorías dogmáticas de la Teoría del Delito, toca su valoración como elemento integrador de la culpabilidad, que al decir de Muñoz (2012) en su obra Derecho Penal Parte General:

La norma penal sólo puede motivar al individuo en la medida en que este pueda conocer, a grandes rasgos, el contenido de sus prohibiciones. $\mathrm{Si}$ el sujeto no sabe que su hacer está prohibido, no tiene ninguna razón para abstenerse de su realización; la norma no le motiva y su infracción, si bien es típica y antijurídica, no puede atribuirse a título de culpabilidad.

Cabe distinguir de lo anterior, como expresa García (2014), en su obra Código Orgánico Integral Penal Comentado que:

Debe aclararse que el objeto de la conciencia de la ilicitud no es el conocimiento de la proposición jurídica infringida o de la punibilidad del hecho, sino, es más bien suficiente con el autor sepa que su comportamiento contradice las exigencias del orden comunitario y por esto, se encuentra jurídicamente prohibido. 
Por consiguiente, la formulación de la culpabilidad desde su concepción normativa en el Código Orgánico Integral Penal, según García (2014), en su obra comentada, se fundamenta en: "(..........) la concurrencia de la responsabilidad penal, la Imputabilidad del autor y el Conocimiento de la Antijuridicidad del Actuar con el que ha obrado". Partiendo de lo planteado, el articulo tiene por objetivo generar una revisión documental sobre la culpabilidad de las personas naturales en el Código Orgánico Integral Penal Ecuatoriano.

\section{MÉTODO}

Se generó una revisión documental sobre el tema abordado, lo cual permite actualizar el estado del arte referente, lo cual contribuye en la articulación de un cuerpo teórico referencial de ser tenido en cuenta por otros investigadores, para lo cual fue preciso desarrollar un proceso de búsqueda, indagación, interpretación, con el fin de construir resultados en concordancia a la inquietud investigativa de la investigadora, sustentada esta postura desde la visión metodológica de Palella Stracuzzi y Martins Pestana (2012).

\section{RESULTADOS}

\section{Causas de Inculpabilidad}

La culpabilidad se caracteriza por presentar un contenido que legitima y limita la imposición de una pena, realmente esta categoría dogmática unida a la de la antijuridicidad, es reconocida como uno de los aspectos más sobresalientes del Derecho Penal, como ciencia, siendo determinante la distinción entre ambas categorías y en consecuencia la diferenciación entre causas de justificación y causas de exclusión de la culpabilidad.

Según Muñoz (2012) en su obra Derecho Penal Parte General:

Actúa antijurídicamente quien sin estar autorizado, realiza un tipo jurídico-penal y ataca con ello un bien jurídico penalmente protegido. Actúa culpablemente quien comete un acto antijurídico tipificado en la ley penal como delito, pudiendo actuar de modo distinto, es decir, conforme a Derecho 
De ello se trasluce el juicio de reproche, como posición que se asume en el Código Orgánico Integral Penal, desde el escenario de la culpabilidad en el Derecho Penal ecuatoriano, junto a los demás elementos que bajo la denominación de triada, valoramos anteriormente.

Las causas de inculpabilidad, constituyen el reverso de la culpabilidad, y lógicamente estas dependerán del concepto que se haya asumido de culpabilidad, tal y como anteriormente comentamos, respecto a los elementos planteados en el artículo 34 de la norma penal en su parte sustantiva. Surge entonces la necesidad de definir su naturaleza, puramente personal, pues estas son solo referidas al sujeto que actúa en el ámbito delictivo, es decir, dependen de la aptitud psíquica del sujeto en relación con el hecho.

Es así que se establecen como causas de exclusión de la culpabilidad; el trastorno mental, es decir la perturbación mental patológica; la edad cronológica, como una realidad biológica; y el estado de embriaguez o intoxicación, de lo cual constituye una excepción los casos de tráfico rodado, es decir de hechos culposos en materia de tránsito.

\section{Trastorno mental}

El artículo 35 del Código Orgánico Integral Penal, exonera de responsabilidad penal a la persona que presentando un trastorno mental debidamente comprobado, realice una conducta de las prohibidas en la propia ley y que se encuentra bajo la conminación de una sanción penal, lo que se justifica en que dicho sujeto al momento de la comisión u omisión, no posee la capacidad de comprender el alcance de su conducta y dirigir ésta conforme a dicho conocimiento.

La perturbación intelectual y volitiva, requieren en la práctica judicial de una ausencia total de dicha capacidad, de ello depende la imputabilidad o inimputabilidad del sujeto, empero dicha perturbación, definida por el legislador ecuatoriano en los artículo 35 y 36, primer párrafo, del Código Orgánico Integral Penal, en el término de trastorno mental, como ya apuntamos, requiere de su debida comprobación, perteneciendo el estudio técnico de la materia al ámbito de la Psiquiatría, convirtiéndose esta ciencia como auxiliadora en la determinación de la 
capacidad intelectual del individuo, y del grado de trastorno mental; a fin de la aplicabilidad de dicha causa de exculpación.

Es significativo que al plantearse la exclusión de culpabilidad, en el sujeto inimputable por trastorno mental, se conmine en la propia norma al juzgador ecuatoriano a dictar medida de seguridad, es decir se excluye la pena como consecuencia jurídica, pero no obstante, se fija una consecuencia en el ámbito jurídico penal, que si bien no tiene un carácter represivo, su cualidad se ubica en el tipo de la medida de seguridad, prevista en el artículo 76 del Código Orgánico Integral Penal, que consiste en el internamiento en centro especializado del inimputable, es decir, en un hospital psiquiátrico, y que tiene una finalidad terapéutica, en cuanto a la superación de la perturbación y la inclusión social del sujeto.

Es decir, si bien la inimputabilidad excluye la responsabilidad penal, siendo una exención de la pena, no obsta a la posibilidad de imposición de medida de seguridad, en el caso de trastorno mental; lo que cumplen una función de control en lugar de las penas. El trastorno mental o enfermedad mental como en muchas otras codificaciones suele llamarse, no necesariamente debe ser absoluta, si bien es cierto que es solamente en esos casos en los que se declara la no responsabilidad penal, dicha causa de exculpación, puede existir tan solo de forma disminuida, lo que por el contrario ella no tendrá efectos de exclusión respecto a la responsabilidad penal, pero sí de mitigación de la pena, en virtud de regla adecuativa de la sanción. Dicha imputabilidad disminuida o semiimputabilidad, es reconocida en el Código Orgánico Integral Penal en su artículo 36 segundo párrafo.

\section{La edad}

Para el Derecho Penal, sin que ello constituya una excepción, la edad es un factor sumamente relevante, pues representa el progresivo avance de las facultades psíquicas del sujeto, por lo que ello hace imprescindible el declarar la edad a partir de la cual pueda reclamarse responsabilidad penal, es decir definir a partir de cuándo, el sujeto se halle en condiciones de responder penalmente y asumir las consecuencias que para sus actos se ha deparado por el Derecho Penal. 
Esa previsión jurídica de la edad penal en el Derecho Penal ecuatoriano, se establece en el artículo 38 del Código Orgánico Integral Penal y está determinada en los 18 años de edad, definiéndose en tal sentido según García (2014) en su obra Código Orgánico Integral Penal, lo siguiente:

Un primer elemento de la capacidad de culpabilidad, tiene que ver con la edad del sujeto, ya que la maduración psíquica y biológica puede influir en la realización de los actos. El injusto del hecho debe ser comprendido en su cabalidad, por ello, el desarrollo moral y mental del menor es un factor importante para conocer el nivel de comprensión de su actuar y no únicamente de sus resultados morales.

Es decir, la plena imputabilidad y responsabilidad no se alcanza con arreglo a la normativa penal en el Ecuador hasta los 18 años de edad.

\section{Responsabilidad penal en casos de embriaguez o intoxicación.}

El efecto psicológico que tiene lugar en los estados de embriaguez o intoxicación, también han resultado de interés desde el escenario de la culpabilidad para el legislador ecuatoriano, al considerarse que la perturbación de las facultades intelectuales o volitivas que de ellos provienen, inciden en esa capacidad de culpabilidad. Al hablar de embriaguez, habría que valorar la misma en el tipo de la voluntaria simple, la culposa, la fortuita, y la peordenada, siendo esta última, la que se provoca para cometer un delito determinado.

El artículo 37 del Código Orgánico Integral Penal, establece la responsabilidad penal en casos de embriaguez e intoxicación, y específicamente en el numeral 1, se establece como causa de exculpación, que el autor en el momento que comete el acto, se halle privado de conocimiento, pero dicha inculpabilidad no termina bajo esa simple formulación dispositiva, sino que hace depender dicha situación del "caso fortuito"; es decir, la causa de exclusión total de la culpabilidad que se enuncia, debe derivar de caso fortuito, o lo que es lo mismo, el agente presenta abolido el conocimiento de su acto, porque se encuentra en estado de embriaguez 0 intoxicación plena, pero ello, reiteramos, a causa de caso fortuito.

En un segundo nivel, numeral 2 ibidem, la cuestión de caso fortuito continúa siendo un determinante, pues vuelve a condicionarse el problema de graduación del efecto psicológico, esta vez, aunque solo disminuido respecto a la alteración no plena de 
las facultades intelectivas o volitivas. Es decir, tal y como manifiesta García (2014), en su obra Código Orgánico Integral Penal Comentado:

En el COIP la ebriedad excluye la imputabilidad solo si es debida acaso fortuito o fuerza mayor, esto es la que se produce sin voluntad o imprudencia por parte del sujeto, es decir aquella cuya producción el sujeto no quiso no previó, ni pudo prever.

Por lo que aun e importante la intensidad del grado de alcohol, no toda embriaguez o intoxicación plena, eximirá de responsabilidad penal, sino solamente la embriaguez fortuita, que lógicamente no es atribuible a imprudencia alguna, o en circunstancias tales que fuera posible prever que daría lugar a cometer el hecho.

Es significativo que aun y tratado desde el prisma de la culpabilidad, expresamente se prevén dos momentos, que no constituyen exclusión de la responsabilidad penal, es decir, el sujeto en dichos casos se hallará apto para soportar o asumir las consecuencias jurídicas penales previstas, y que penden de su conducta penalmente relevante.

De esa forma el numeral 3 del artículo 37 del Código Orgánico Integral Penal, expresa de forma categórica que si el estado de embriaguez o de intoxicación no proviene de caso fortuito, ello no trasciende a la exclusión de responsabilidad penal, ni a su atenuación; refiriéndose así, a embriaguez voluntaria simple y a la embriaguez culposa. En igual sentido el numeral 4 ibídem, referido a la embriaguez preordenada, prevé el supuesto de que a dicho estado se llegue de forma premeditada, con el fin de cometer la infracción o justificar la misma, situación que eleva a la categoría de circunstancia agravante.

\section{Punibilidad en los casos de no exclusión de responsabilidad penal}

Como se trata anteriormente, bajo la institución de la culpabilidad como categoría dogmática de Derecho Penal, no solamente se trata el tema de las causas de inculpabilidad, ligadas a la inimputabilidad y desconocimiento de la antijuridicidad de la conducta, sino también se establecen los casos en que dichas causas de exclusión, deviene de forma incompleta y no total, y en consecuencia no son excluyentes, pero si atenuantes de dicha responsabilidad penal, desde el escenario mismo de la punición y consecuencias penológicas que se establecen. Es así que el beneficio de régimen de atenuación de pena que se establece en ambos casos 
Iustitia Socialis. Revista Arbitrada de Ciencias Jurídicas.

Año IV. Vol. IV. N 1 . Edición Especial 2019

Hecho el depósito de Ley: FA2016000064 ISSN: 2542-3371

FUNDACIÓN KOINONIA (F.K). Santa Ana de Coro, Venezuela

Tania Muñoa Vidal

consiste en la atenuación de un tercio de la pena mínima prevista para el tipo penal calificado.

\section{REFERENCIAS CONSULTADAS}

1. Código Orgánico Integral Penal. Decreto Legislativo. Registro Oficial 180 del 10 de febrero de 2014

2. Constitución de la República del Ecuador. Decreto Legislativo 0. Registro Oficial 449 del 20 de octubre de 2008.

3. García, Falcones. (2014).Código Orgánico Integra Penal Comentado. Tomo I, II. Quito: Latitud Cero Editores

4. Mir, Santiago. (2011). Derecho Penal Parte General. Barcelona: Reppertor

5. Muñoz, Francisco. (2000). Presente y futuro de la dogmática jurídico-penal. Valencia

6. Muñoz, Francisco. (2009). Cuestiones dogmáticas básicas en los delitos económicos. Valencia

7. Muñoz, Francisco. (2012). Derecho Penal Parte General. Valencia: Tirant lo Blanch

8. Palella Stracuzzi, S. \& Martins Pestana, F. (2012). Metodología de la investigación cuantitativa. Fondo editorial de la Universidad Pedagógica Libertador. Caracas, Venezuela.

9. Quirós, René. (2006). Manual de Derecho Penal. Tomo I,II,III. La Habana: Félix Varela

10. Roxin, C., \& Vásquez, M. A. A. (2007). La teoría del delito en la discusión actual. Grijley.

\section{REFERENCES CONSULTED}

1. Organic Integral Criminal Code. Legislative Decree, Official Register 180 of February 10, 2014.

2. Constitution of the Republic of Ecuador. Legislative Decree 0. Official Register 449 of October 20, 2008. 
Iustitia Socialis. Revista Arbitrada de Ciencias Jurídicas.

Año IV. Vol. IV. N 1 . Edición Especial 2019

Hecho el depósito de Ley: FA2016000064 ISSN: $2542-3371$

FUNDACIÓN KOINONIA (F.K). Santa Ana de Coro, Venezuela

Tania Muñoa Vidal

3. Garcia, Falcones. (2014) Integral Organic Code Criminal, Volume I, II. Quito: Latitude Zero Editors.

4. Mir, Santiago. (2011) Criminal Law General Part. Barcelona: Repeater

5. Muñoz, Francisco. (2000) Present and future of legal-criminal dogmatics. Valencia.

6. Muñoz, Francisco. (2009). Basic dogmatic issues in economic crimes. Valencia.

7. Muñoz, Francisco. (2012). Criminal Law General Part. Valencia: Tirant lo Blanch.

8. Palella Stracuzzi, S. \& Martins Pestana, F. (2012). Quantitative research methodology. Editorial Fund of the Libertador Pedagogical University. Caracas Venezuela.

9. Quiros, René. (2006) Criminal Law Manual. Volume I, II, III. Havana: Félix Varela.

10. Roxin, C. and Vásquez, M. A. A. (2007). The theory of crime in the current discussion. Grijley.

(C2019 por los autores. Este artículo es de acceso abierto y distribuido según los términos y condiciones de la licencia Creative Commons Atribución-NoComercial-Compartirlgual 4.0 Internacional (CC BY-NC-SA 4.0) (https://creativecommons.org/licenses/by-nc-sa/4.0/). 